\title{
Fast Radio Bursts - implications and future prospects for Fermi
}

\author{
Manisha Caleb* \\ Jodrell Bank Centre for Astrophysics, School of Physics and Astronomy, University of \\ Manchester, Manchester, M13 9PL, UK \\ E-mail: manisha.caleb@manchester.ac.uk
}

\begin{abstract}
The recent development of sensitive, high time resolution instruments at radio telescopes has enabled the discovery of millisecond duration fast radio bursts (FRBs). The FRB class encompasses a number of single pulses, many unique in their own way, so far hindering the development of a consensus for their origin. The key to demystifying FRBs lies in discovering many of them in realtime in order to localise them and identity commonalities. Despite rigorous follow-up, only one FRB has been seen to repeat suggesting the possibility of there being two independent classes of FRBs and thus two classes of possible progenitors. This paper discusses recent developments in the field, the FRB-GRB connection, some of the open questions in FRB astronomy and how the next generation telescopes are vital in the quest to understand this enigmatic population.
\end{abstract}

7th Fermi Symposium 2017

15-20 October 2017

Garmisch-Partenkirchen, Germany

\footnotetext{
* Speaker.
} 


\section{Introduction}

Time domain radio astronomy has recently discovered a new class of radio transient called Fast Radio Bursts (FRBs). This class comprises bright, millisecond duration ( $\sim$ Jy) single pulses, mostly of singular occurrence, exhibiting a quadratic frequency-dependent time delay (called dispersion measure), consistent with propagation through a cold ionized plasma. The dispersion measure (DM) is the integrated free electron density along the line-of-sight given by,

$$
\mathrm{DM}=\int_{0}^{d} n_{e} d l
$$

and can be approximated to be a proxy for distance. For example, the DMs of pulsars in our Galaxy have been used to build a model of the electron density distributions through various lines-of-sight $[1,2]$.

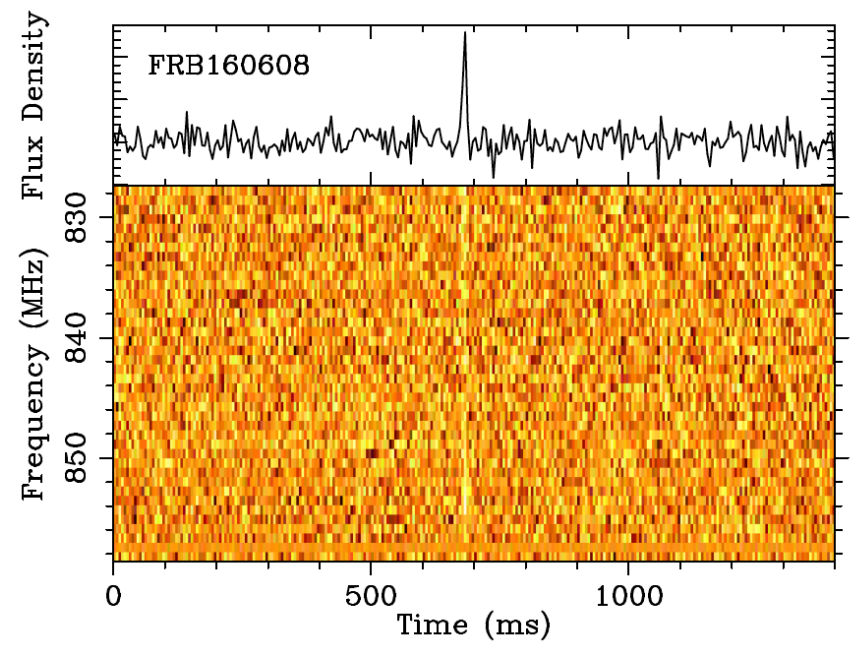

Figure 1: Time vs frequency behaviour of FRB 160410 detected at the UTMOST. The top panel shows the time averaged profile and the bottom plot shows the frequency profile after the effect of dispersion has been accounted for resulting in a width of $7.4 \mathrm{~ms}$. Figure taken from [3].

The discovery of the sporadically emitting rotating radio transients in 2006 [4] motivated astronomers to search for more such similar pulses resulting in the discovery of the prototypical FRB dubbed the 'Lorimer burst', in 2007 [5]. The standout feature of the Lorimer burst was its dispersion measure, which was well in excess of the Galactic contribution for the observed line-of-sight. This is also the primary distinguishing feature between rotating radio transients and FRBs [6]. The redshift inferred from the excess DM, using a simple empirical scaling relation in [7] and [8] from gamma-ray bursts (GRBs), implied an extragalactic if not cosmological ( $\sim \mathrm{Gpc}$ ) origin for the Lorimer burst. The inferred redshift is an upper limit, as the interstellar medium (ISM) of the host galaxy and environment local to the progenitor would also contribute to the DM. The high brightness temperatures of FRBs $\left(\mathrm{T}_{\mathrm{b}}>10^{35} \mathrm{~K}\right)$ strongly suggest a coherent emission mechanism [9]. If FRBs are indeed coherent emitters at cosmological distances, they could open up a whole new window to probe the extragalactic and even distant Universe. They could potentially be used to solve the case of the 'missing baryons' in the Universe [10], map the 
intergalactic magnetic field [11] and even acquire an independent measure of the dark energy equation of state [12].

More than 30 FRBs have been discovered since 2007, with various telescopes (Parkes, Green Bank Telescope, Arecibo, UTMOST, ASKAP) over a range of frequencies (1.4 GHz, $800 \mathrm{MHz}, 2$ $\mathrm{GHz}, 4-8 \mathrm{GHz}$ ), of which only $24 \mathrm{FRBs}$ have been published (see [13] for references). However only one of these 24 FRBs, the one discovered at the Arecibo observatory, has been seen to repeat, despite telescopes having spent several hundreds of hours re-observing the positions of known FRBs [14]. Leading progenitor models for FRBs range from binary neutron star mergers (e.g. [15]) and the collapse of a supramassive neutron star into a black hole [16] in the cataclysmic scenario, to giant flares from young energetic magnetars in supernova remnants (e.g. [17]) and extragalactic pulsars in nearby galaxies [18] in the repeating scenario. Given the light travel-time and the fact that FRBs typically last only a few milliseconds, the radius of the source producing it would be $\sim$ few hundred kms implying a small emission region size. This would not be the case though, if for example, FRBs were to arise at the intersection of a supernova remnant shock wave and progenitor source wind bubble [19].

\section{Open questions in FRB astronomy}

It has been a decade since the discovery of FRBs and despite the rapid and significant development in the field, no consensus has emerged regarding their origin. Despite all the single pulses being broadly classified as FRBs, no two pulses are the same. In order to better understand the population, it is evident that we need a larger sample with arcsecond localisation and preferably a counterpart at another wavelength.

\subsection{Are there two (or more) classes of FRBs?}

The repeating FRB discovered at the Arecibo observatory is indicative of a type of progenitor that is not destroyed by the energetic events producing these FRBs. The repeat pulses have been used to localise the source to a low-metallicity dwarf galaxy at $z \sim 0.19273(8)$ [20]. This is the only FRB to have been unambiguously localised to a host. The pulses appear to be highly clustered in time over the course of a couple of years with no underlying periodicity, and DMs consistent to within the uncertainties [21]. This is strongly suggestive of a single astronomical object being responsible for these bursts. No two pulses from the repeater look the same and they exhibit spectral cut-offs, frequency structure and wildly varying spectral indices similar to the giant pulses from the Crab pulsar. An interesting property of these pulses is their lack of obvious pulse broadening due to possible multi-path scattering upon interaction with turbulent plasma along the path of propagation, quite commonly seen in pulsars. This suggests that the observed widths of $\sim 3-9 \mathrm{~ms}$ are quite possibly their intrinsic widths [22].

The fact that none of the other FRBs have been seen to repeat yet, does not rule out the possibility of a non-cataclysmic progenitor. Most of the non-repeating FRBs have been discovered at the Parkes radio telescope whose sensitivity is $\sim 10$ times less than the Arecibo telescope. Some of these pulses show spectral cut-offs and frequency structures similar to the repeater [23, 24]. The simultaneous detection of FRB 150418 with the Parkes radio telescope at $1.4 \mathrm{GHz}$ and nondetection of the burst with the Murchison Widefield Array at $150 \mathrm{MHz}$, placed a limit of $\alpha \gtrsim-3.0$, 


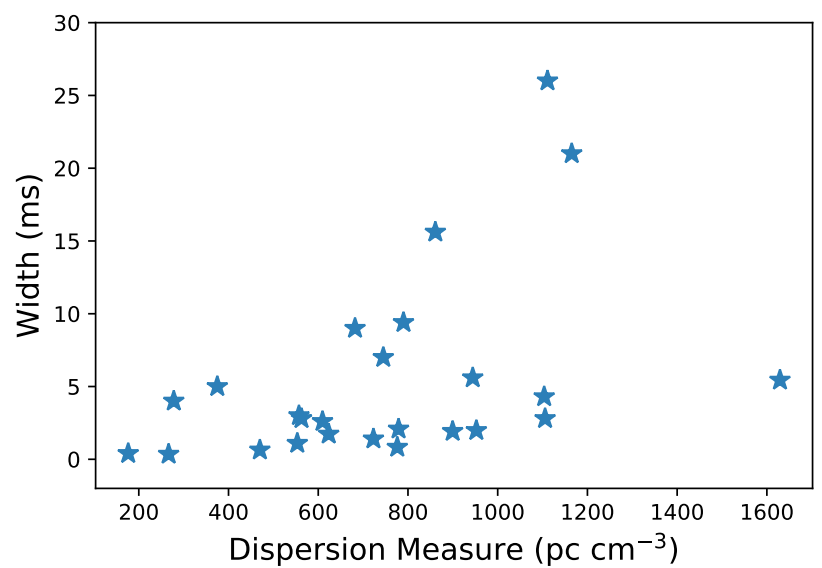

Figure 2: The relstionship between pulse width and DM. For the FRBs published, the pulse width is thought to be the result of Galactic (which is typically very small) and non-galactic (IGM + host galaxy + progenitor) contributions. If the scattering originates in the homogenous and diffuse IGM through which all FRBs propagate, all the pulses would be broadened, which is not the case. This suggests that the IGM is not responsible for the observed width making the host galaxy ISM and progenitor environment strong candidates.

Table 1: Summary of the differences between the repeating and non-repeating FRBs.

\begin{tabular}{|l|l|l|}
\hline Parameter & Repeater (FRB 121102) & Non-repeating FRBs \\
\hline Discovery telescope & Arecibo & Parkes, GBT, UTMOST, ASKAP \\
\hline Detection telescope & GBT, VLA, Effelsberg & - \\
\hline Frequencies $(\mathrm{GHz})$ & $1.4,2,4-8$ & $1.4,0.8,0.843$ \\
\hline Spectral index & -10 to +15 & $\gtrsim-3.0$ for FRB150418 \\
\hline Localisation & Dwarf galaxy at $z \sim 0.19273(8)$ & No localisation yet \\
\hline Polarisation & No detectable polarisation & Varied polarisation with no trend \\
\hline Widths & $3-9 \mathrm{~ms}$ & $\lesssim 0.4-26 \mathrm{~ms}$ \\
\hline Scattering & No & Measured for some FRBs \\
\hline Frequency structure & Yes & Visible in some FRBs \\
\hline Periodicity & No underlying periodicity & Singular events \\
\hline DM variation & Consistent to within uncertainties & - \\
\hline
\end{tabular}

on its spectral index [25]. Certain non-repeating FRB pulses do show strong evidence of multi-path scattering which when accounted for, imply much narrower intrinsic widths $(<0.4-4 \mathrm{~ms})$ when compared to the repeater.

Figure 2 shows the relationship between pulse width and DM. The width of an FRB is the sum of contributions of Galactic and non-Galactic components. Pulsars at Galactic latitudes similar to FRBs exhibit orders of magnitude smaller scattering timescales than FRBs [26, 27]. The nonGalactic contributions could arise from the host galaxy and the intergalactic medium (IGM). In their 
empirical scaling relation between DM and scattering, [28] show that the IGM's contribution to the pulse broadening is orders of magnitude less than the Milky Way's ISM. The FRB with the greatest DM would be expected to have the broadest pulse width, which is evidently not the case from Figure 2. Along with the fact that both time resolved and time unresolved pulses exist, this suggests that the IGM through which all the FRBs traverse, is not responsible for the pulse broadening, which makes the host galaxy and the progenitor circumburst medium strong candidates.

In the simplest case, the repeater could belong to a different evolutionary phase of a given source population with a different $\log N-\log \mathscr{F}$ compared to the non-repeating FRBs. The sensitivity of the Parkes radio telescope could constrain us from detecting the very faint pulses resulting in only the bright tail end of the pulse energy distribution being visible, leading to the detection of oneoff events. This is supported by the fact that even the published brightest pulse from the repeater would just be detectable above the threshold at Parkes. Or FRBs could simply have multiple subpopulations, similar to GRBs.

\subsection{Emission in other wavebands? What are the prospects for Fermi?}

Of the 24 published FRBs, only the real-time detections have allowed for prompt follow-up at other wavelengths. Whether FRBs produce prompt signatures at other wavelengths still remains a mystery. Creating opportunities for follow-up and simultaneous observations with existing radio telescopes, high-energy and gravitational wave observatories, in anticipation of the large sample of FRBs expected with next generation radio telescope could prove vital to progress in the field. We can expect breakthroughs in our comprehension of FRBs by this approach, as was demonstrated in the case of GRBs and the repeating FRB 121102. The association of this FRB with a dwarf galaxy will potentially influence future follow-up strategies. An association of an FRB with a counterpart at another wavelength would not only provide valuable insights regarding their progenitors but also their emission mechanisms. However this type of multi-wavelength detection of FRBs at different wavelengths is highly uncertain as only one FRB has been seen to repeat. Commensal observing and shadowing similar to the strategy followed by the SUPERB collaboration [29], offer great potential for the identification of an FRB progenitor. Most progenitor models for repeating FRBs do not predict emission at high energies as the progenitors are pulsar-like whose emission is typically restricted to the radio spectrum [30]. Cataclysmic progenitors however do predict high energy emission in the form of gamma-rays, X-rays and optical afterglows [15].

It was recently reported that a gamma-ray transient detected with the burst alert telescope instrument on the SWIFT observatory was coincident with FRB 131102 with an association significance of $3.2 \sigma$ [31]. The association claim was however refuted [32] based on the non-detection of a radio afterglow at the location of the gamma-ray transient and the discovery of an AGN both temporally and spatially coincident with the FRB. Data taken with the Fermi gamma-ray burst monitor was searched for gamma-ray bursts associated with the repeating FRB 121102 during the time it was visible to the Fermi sky [22]. For the 4 radio bursts that were analysed, the corresponding gamma-ray data was found to be consistent with the persistent GBM background level. Based on the measured luminosity distance this corresponds to a $10-100 \mathrm{keV}$ burst energy limit of $5 \times 10^{47} \mathrm{erg}$.

A targeted search for prompt radio emission from GRBs at $1.4 \mathrm{GHz}$ was undertaken by [33] using a 12-m telescope to slew automatically to the GRB coordinates based on GCN alerts. They 
report the detection of 2 radio pulses, 524 and 1076 seconds after the GRB, with S/N $>6 \sigma$ and DMs greater than the Galactic contribution. However they calculate a probability of only $2 \%$ association with the corresponding GRBs based on simple population arguments. [34] performed an experiment similar to [33]. They observed five GRBs using a 26-m radio telescope, automated to respond to GCN alerts and slew to the source coordinates within minutes. Non-detections of any significant radio pulses $>5 \sigma$ in their experiment, agree with the lack of consistency between the FRB and GRB event rates presented in [35] $\left(R_{\mathrm{FRB}} \sim 10^{-3} \mathrm{gal}^{-1} \mathrm{yr}^{-1}>R_{\mathrm{GRB}} \sim 10^{-6} \mathrm{gal}^{-1} \mathrm{yr}^{-1}\right)$.

\subsection{Can we probe cosmic magnetism?}

Faraday rotation of polarised sources have proven to be powerful probes of the magnetic field; both at the source and in the ISM. As the radio wave propagates from source to observer, the plane of polarisation of linearly polarised light is rotated under the influence of a magnetic field with the magnitude of rotation quantified by the rotation measure (RM). The RM due to a source at cosmological distances is given by,

$$
\operatorname{RM}(z)=\frac{e^{3}}{2 \pi m_{\mathrm{e}}^{2} c^{4}} \int_{0}^{z} \frac{n_{e}(z) B_{\|}(z)}{(1+z)^{2}} \cdot \frac{d l}{d z} d z \quad \operatorname{radm}^{2}
$$

where $n_{\mathrm{e}}$ is the electron density in particles per cubic metre, $B_{\|}$is the vector magnetic field parallel to the line-of-sight in microgauss, $d l$ is the elemental vector towards the observer along the line-of-sight and $(1+z)$ is due to the redshifting of the observed frequencies. A combination of the RM and DM can be used to measure the integrated magnetic field along the line-of-sight. If FRBs are cosmological in nature, their polarisation properties could provide the first measurements of the intergalactic magnetic field and also insight into the magnetic fields associated with the progenitor. Unlike the published pulses from the repeating FRB which show no polarisation, some non-repeating FRBs do show significant polarisation, though with considerable variation. Of the 24 FRBs, 7 have full polarisation information of which 5 are published. Though FRBs 140514 [36] and 150807 [37] discovered at the Parkes radio telescope are highly linearly polarised, their RM values are consistent with Milky Way's foreground contribution for the given lines-of-sight implying a negligible or zero contribution from the host galaxy and progenitor. In the host galaxy and progenitor, this is indicative of either low ordered magnetic fields or disordered magnetic fields which cancel each other resulting in no net RM value. The contribution from the IGM is typically of the order nano-Gauss [38] and negligible compared to the contributions from the Galactic foreground and potential host galaxy and progenitor. In contrast are FRBs 110523 [39] and 160102 (Caleb et al., in prep) which have significant linear polarisation and RM values well in excess of the Galactic foreground contribution suggesting a highly magnetised medium and ordered magnetic fields in the host galaxy or immediate vicinity of the progenitor. The small sample size and lack of trend makes it difficult to provide any explanation for FRB emission mechanisms and properties to date.

\section{Summary and future prospects}

FRBs are one of the most tantalizing topics in astronomy of the last decade. We presently know of 36 FRBs of which only one is seen to repeat, but with no discernible periodicity. The 
large sample of non-repeating FRBs is quite diverse with pulses that exhibit frequency structure, spectral cut-offs, are time resolved, time unresolved, linearly polarised, circularly polarised and unpolarised. Multi-element interferometric detections of FRBs in the radio with instant localisation is the future. We can expect localisation to a few arcseconds radius with the next generation radio telescopes like MeerKAT, UTMOST, Westerbork radio telescope, Australian Square Kilometre Array Pathfinder (ASKAP), Canadian Hydrogen Intensity Mapping Experiment (CHIME) and DSA-10, coming online. The UTMOST-2D project is a planned upgrade to the UTMOST telescope to provide better spatial localisation of a few arcseconds radius. The ASKAP and Westerbork telescopes with their phased array feed technologies make them excellent instruments for FRB host localisations. The CHIME cylindrical telescope with its massive field-of-view of $250 \mathrm{deg}^{2}$ is expected to detect tens of FRBs per day though localisation will be relatively poor ( $\sim$ arcmin). The MeerKAT pathfinder to the SKA is also expected to provide near instantaneous localisation of a few arcseconds radius. The MeerTRAP project at MeerKAT will undertake high time resolution, fully commensal transient searches in parallel with most of the legacy science project observations. This is particularly advantageous for FRB searches as it will allow a large sky coverage, multiple visits to the same field to search for repeats, and variable cadence observations.

These future radio telescopes will enable unambiguous association of an FRB with a host galaxy even in the case of non-repeating bursts. Rapid follow-up and high cadence observations at $\mathrm{X}$-ray, optical and gamma-ray energies accompanying the localisation in the radio is required to robustly sample any lightcurve of a transient afterglow. The current limits are insufficiently stringent to constrain the FRB-GRB connection. However future radio telescopes along with sensitive observations with Fermi and the Cerenkov Telescope Array might be able to constrain the connection. We can expect the next decade to answer some if not all the currently open questions in FRB astronomy.

\section{Acknowledgements}

This project has received funding from the European Research Council (ERC) under the European Union's Horizon 2020 research and innovation programme (grant agreement No 694745). Parts of this research was funded by the Australian Research Council Centre for All-Sky Astrophysics (CAASTRO), through project number CE110001020, and the Laureate Fellowship FL150100148.

\section{References}

[1] J. M. Cordes and T. J. W. Lazio, NE2001.I. A New Model for the Galactic Distribution of Free Electrons and its Fluctuations, ArXiv Astrophysics e-prints (July, 2002), [astro-ph/0207156].

[2] J. M. Yao, R. N. Manchester and N. Wang, A New Electron-density Model for Estimation of Pulsar and FRB Distances, 835 (Jan., 2017) 29, [1610 . 09448].

[3] M. Caleb, C. Flynn, M. Bailes, E. D. Barr, T. Bateman, S. Bhandari et al., The first interferometric detections of fast radio bursts, 468 (July, 2017) 3746-3756, [1703.10173]. 
[4] M. A. McLaughlin, A. G. Lyne, D. R. Lorimer, M. Kramer, A. J. Faulkner, R. N. Manchester et al., Transient radio bursts from rotating neutron stars, 439 (Feb., 2006) 817-820, [astro-ph/0511587].

[5] D. R. Lorimer, M. Bailes, M. A. McLaughlin, D. J. Narkevic and F. Crawford, A bright millisecond radio burst of extragalactic origin, 318 (2007) 777-, [0 709.4301$].$

[6] E. F. Keane, Classifying RRATs and FRBs, 459 (June, 2016) 1360-1362, [1512.02513].

[7] K. Ioka, The cosmic dispersion measure from gamma-ray burst afterglows: Probing the reionization history and the burst environment, 598 (Dec., 2003) L79-L82, [astro-ph/ 0309200 ].

[8] S. Inoue, Probing the cosmic reionization history and local environment of gamma-ray bursts through radio dispersion, 348 (Mar., 2004) 999-1008, [a stro-ph/ 0309364 ].

[9] J. Luan and P. Goldreich, Physical Constraints on Fast Radio Bursts, 785 (Apr., 2014) L26, [1401.1795].

[10] M. McQuinn, Locating the "Missing” Baryons with Extragalactic Dispersion Measure Estimates, 780 (jan, 2014) L33, [1309.4451].

[11] Z. Zheng, E. O. Ofek, S. R. Kulkarni, J. D. Neill and M. Juric, Probing the Intergalactic Medium with Fast Radio Bursts, 797 (dec, 2014) 71, [1409.3244].

[12] B. Zhou, X. Li, T. Wang, Y.-Z. Fan and D.-M. Wei, Fast radio bursts as a cosmic probe?, 89 (may, 2014) 107303, [1401.2927].

[13] E. Petroff, E. D. Barr, A. Jameson, E. F. Keane, M. Bailes, M. Kramer et al., FRBCAT: The Fast Radio Burst Catalogue, 33 (Sept., 2016) e045, [1601.03547].

[14] A. Rane and D. Lorimer, Fast Radio Bursts, Journal of Astrophysics and Astronomy 38 (Sept., 2017) 55.

[15] T. Totani, Cosmological Fast Radio Bursts from Binary Neutron Star Mergers, 65 (oct, 2013) L12, [1307.4985].

[16] H. Falcke and L. Rezzolla, Fast radio bursts: the last sign of supramassive neutron stars, 562 (Feb., 2014) A137, [1307.1409].

[17] Y. Lyubarsky, A model for fast extragalactic radio bursts, 442 (July, 2014) L9-L13, [1401. 6674$].$

[18] J. M. Cordes and I. Wasserman, Supergiant pulses from extragalactic neutron stars, 457 (Mar., 2016) 232-257, [1501.00753].

[19] B. D. Metzger, E. Berger and B. Margalit, Millisecond Magnetar Birth Connects FRB 121102 to Superluminous Supernovae and Long-duration Gamma-Ray Bursts, 841 (May, 2017) 14, [1701.02370].

[20] S. P. Tendulkar, C. G. Bassa, J. M. Cordes, G. C. Bower, C. J. Law, S. Chatterjee et al., The Host Galaxy and Redshift of the Repeating Fast Radio Burst FRB 121102, 834 (Jan., 2017) L7, [1701.01100].

[21] L. G. Spitler, P. Scholz, J. W. T. Hessels, S. Bogdanov, A. Brazier, F. Camilo et al., A repeating fast radio burst, 531 (Mar., 2016) 202-205, [1603.00581].

[22] P. Scholz, S. Bogdanov, J. W. T. Hessels, R. S. Lynch, L. G. Spitler, C. G. Bassa et al., Simultaneous X-Ray, Gamma-Ray, and Radio Observations of the Repeating Fast Radio Burst FRB 121102, 846 (Sept., 2017) 80, [1705.07824]. 
[23] D. J. Champion, E. Petroff, M. Kramer, M. J. Keith, M. Bailes, E. D. Barr et al., Five new fast radio bursts from the HTRU high-latitude survey at Parkes: first evidence for two-component bursts, 460 (July, 2016) L30-L34, [1511.07746].

[24] W. Farah, M. Bailes, A. Jameson, C. Flynn, I. Andreoni, T. Bateman et al., Detection of a highly scattered Fast Radio Burst at the Molonglo Radio Telescope, The Astronomer's Telegram 10867 (Oct., 2017).

[25] E. F. Keane, S. Johnston, S. Bhandari, E. Barr, N. D. R. Bhat, M. Burgay et al., The host galaxy of a fast radio burst, 530 (Feb., 2016) 453-456, [1602 .07477].

[26] N. D. R. Bhat, J. M. Cordes, F. Camilo, D. J. Nice and D. R. Lorimer, Multifrequency Observations of Radio Pulse Broadening and Constraints on Interstellar Electron Density Microstructure, 605 (Apr., 2004) 759-783, [astro-ph/0401067].

[27] M. A. Krishnakumar, D. Mitra, A. Naidu, B. C. Joshi and P. K. Manoharan, Scatter Broadening Measurements of 124 Pulsars At 327 Mhz, 804 (May, 2015) 23, [1501.05401].

[28] J.-P. Macquart and J. Y. Koay, Temporal smearing of transient radio sources by the intergalactic medium, 776 (oct, 2013) 125, [1308.4459].

[29] E. F. Keane, E. D. Barr, A. Jameson, V. Morello, M. Caleb, S. Bhandari et al., The SUrvey for Pulsars and Extragalactic Radio Bursts I: Survey Description and Overview, ArXiv e-prints (June, 2017), [1706.04459].

[30] J. M. Cordes, R. S. Wharton, L. G. Spitler, S. Chatterjee and I. Wasserman, Radio Wave Propagation and the Provenance of Fast Radio Bursts, ArXiv e-prints (May, 2016) , [1605.05890].

[31] J. J. DeLaunay, D. B. Fox, K. Murase, P. Mészáros, A. Keivani, C. Messick et al., Discovery of a Transient Gamma-Ray Counterpart to FRB 131104, 832 (Nov., 2016) L1, [1611.03139].

[32] R. M. Shannon and V. Ravi, Radio-interferometric Monitoring of FRB 131104: A Coincident AGN Flare, but No Evidence for a Cosmic Fireball, 837 (Mar., 2017) L22, [1611. 05580].

[33] K. W. Bannister, T. Murphy, B. M. Gaensler and J. E. Reynolds, Limits on Prompt, Dispersed Radio Pulses from Gamma-Ray Bursts, 757 (Sept., 2012) 38, [1207. 6399].

[34] D. Palaniswamy, R. B. Wayth, C. M. Trott, J. N. McCallum, S. J. Tingay and C. Reynolds, A Search for Fast Radio Bursts Associated with Gamma-Ray Bursts, 790 (July, 2014) 63, [14 06.1850 ].

[35] D. Thornton, B. Stappers, M. Bailes, B. Barsdell, S. Bates and Bhat, A population of fast radio bursts at cosmological distances, 341 (July, 2013) 53-56, [1307.1628].

[36] E. Petroff, M. Bailes, E. D. Barr, B. R. Barsdell, N. D. R. Bhat, F. Bian et al., A real-time fast radio burst: polarization detection and multiwavelength follow-up, 447 (Feb., 2015) 246-255, [1412.0342].

[37] V. Ravi, R. M. Shannon, M. Bailes, K. Bannister, S. Bhandari, N. D. R. Bhat et al., The magnetic field and turbulence of the cosmic web measured using a brilliant fast radio burst, Science 354 (Dec., 2016) 1249-1252, [1611.05758].

[38] M. S. Pshirkov, P. G. Tinyakov and F. R. Urban, New Limits on Extragalactic Magnetic Fields from Rotation Measures, Physical Review Letters 116 (May, 2016) 191302, [1504. 06546 ].

[39] K. Masui, H.-H. Lin, J. Sievers, C. J. Anderson, T.-C. Chang, X. Chen et al., Dense magnetized plasma associated with a fast radio burst, 528 (Dec., 2015) 523-525, [1512.00529]. 